\section{A novel technique effective for caliceal diverticular stones}

Current minimally invasive treatments for radiopaque caliceal diverticular calculi include shock wave lithotripsy, ureteroscopy, laparoscopy and percutaneous nephrolithotomy (PNL). Kim et al. have recently reviewed the outcomes for patients undergoing a novel, single-stage PNL technique that eliminates ureteral catheterization and entry into the renal collecting system.

Twenty-one patients with caliceal diverticular calculi took part in the study from February 2001 to September 2003 and were treated with single-stage PNL. Results showed that the mean operative time was reduced to $58.5 \mathrm{~min}$. A stone-free rate of $85.7 \%$ was achievedconfirmed by non-contrast computerized tomography-and 14 out of 16 patients with follow-up excretory urography at 3 months had complete diverticular resolution. No transfusions were necessary and no pulmonary complications occurred as all punctures were performed infracostally. All patients, with the exception of one, were discharged home tubeless on postoperative day 1 .

The authors conclude that standard PNL is the most effective approach for the treatment of symptomatic radiopaque caliceal diverticular stones; however, using a single-stage modified PNL technique significantly reduced operative times, while still achieving diverticular obliteration and a high stone-free rate. The authors highlight that single-stage PNL can be used for all radiopaque diverticular calculi regardless of diverticular location in the kidney.

Original article Kim SC et al. (2005) Percutaneous nephrolithotomy for caliceal diverticular calculi: a novel single stage approach. J Urol 173: 1194-1198

\section{Increased warning time in the management of urinary urgency}

A recent paper by Cardozo and Dixon is one of the first studies to assess the effect of darifenacin-a selective M3-receptor antagonist developed for overactive bladder syndrome-on the warning time associated with urinary urgency.

This multicenter, double-blind, placebocontrolled study included 72 subjects who had had symptoms of urgency for 6 months or greater and four or more episodes of urgency per day. Subjects were randomized in a 1:1 ratio to receive darifenacin ( $30 \mathrm{mg}$ once daily) or placebo for 2 weeks. Electronic event recorders were used to monitor urge-void cycles in a $6 \mathrm{~h}$ period in the clinic, and subjects were instructed in the use of a paper diary and asked to complete this at home.

Warning time (defined as the time from the first sensation of urgency to voluntary incontinence) was significantly longer in the darifenacin-treated patients compared with placebo. Darifenacin-treated patients also achieved a significant decrease in the severity of urgency compared with placebo; however, this was only shown during clinic-centered monitoring periods. Only mild or moderate adverse events were reported.

Limitations of this study included the small number of subjects and short treatment duration. The authors conclude, however, that treatment with darifenacin significantly increased mean, median and minimum warning times compared with placebo. This should confer a decrease in incontinence episodes and, therefore, increase subject confidence. The authors note that future follow-up studies are needed to determine the degree of increase in warning time needed to significantly enhance quality of life in these patients.

Original article Cardozo L and Dixon A (2005) Increased warning time with darifenacin: a new concept in the management of urinary urgency. J Urol 173: 1214-1218

\section{Bladder-neck contracture following surgery for benign prostatic hyperplasia}

Transurethral resection of the prostate (TURP) is regarded as the mainstay treatment of benign prostatic hyperplasia; however, bladder-neck contracture (BNC) has been noted as a complication which often necessitates reoperation. The rate for reoperation with TURP is around $15-20 \%$. Another treatment for benign prostatic hyperplasia is transurethral incision of the prostate (TUIP), which has reoperative rates of around $7-7.6 \%$ but has several disadvantages compared with TURP: it cannot be used in patients with adenoma weight of $>30 \mathrm{~g}$; it is not effective in patients with a prominent median 